\title{
Genetics influences development of AD pathology
}

\begin{abstract}
$c 4$
propagation

of tau and $A \beta$

was associated

with a common

genetic profile

related mainly

to lipid

metabolism

The genetic influence on deposition and propagation of late-onset Alzheimer disease (AD) pathology has become clearer after publication of two new studies. In both, novel techniques have been used to provide insight into disease mechanisms.

In the first study, Jorge Sepulcre and colleagues studied genetic associations with tau and amyloid- $\beta$ $(\mathrm{A} \beta)$ propagation in the brain. These AD pathologies are known to spread through specific brain networks that are vulnerable to this propagation, but the way in which this network-specific vulnerability relates to the genetic risk factors for $\mathrm{AD}$ has been unexplored.

"At present, there are limited in vivo approaches to identify and characterize the relationship between genetic traits and neuroimaging phenotypes of $\mathrm{AD}$ pathology propagation," explains Sepulcre. "We developed a new approach to combine graph theory metrics able to detect in vivo spreading pathways of tau and $A \beta$ deposits, and genetic information in cross-sectional and longitudinal cohorts."

The researchers combined information about tau and $\mathrm{A} \beta$ propagation, obtained with PET imaging in participants in the Havard Aging Brain Study, with data on gene
\end{abstract}

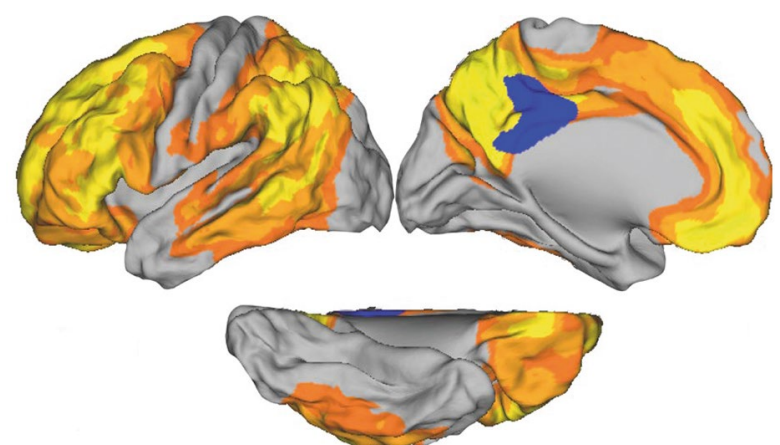

Cortical connectivity map showing prediction of amyloid- $\beta$ propagation from the posterior cingulate cortex (blue). Adapted from Sepulcre, J. et al. Nat. Med. https://doi.org/10.1038/s41591-018-0206-4 (2018), Springer Nature Limited. expression levels in the cortex from the Allen Human Brain Atlas. The combined data revealed colocalization of pathology propagation with expression of specific genes.

Analysis revealed that propagation of tau and $A \beta$ was associated with a common genetic profile related mainly to lipid metabolism. Interactome analysis revealed the genes with central roles in this genetic network, one of which was APOE. Analysis of genes associated with either tau or $\mathrm{A} \beta$ propagation alone revealed an 'axon-related' profile for tau and a 'dendrite-related' profile for $A \beta$.

The novel approach identified distinct genetic profiles that contribute to different aspects of $\mathrm{AD}$ pathology propagation, and Sepulcre and colleagues have more plans to apply their approach. "We will go deeper into this topic by expanding our findings towards individual-level phenotypegenotype associations," says Sepulcre. "We are extremely interested in disentangling relevant features of $\mathrm{AD}$ risk in preclinical stages."

In the second study, Ilyas Kamboh and colleagues did look at the preclinical stages of $\mathrm{AD}$ to identify genetic risk factors for $\mathrm{AD}$. Genes that have been associated with $\mathrm{AD}$ in genome-wide association studies do not explain all $\mathrm{AD}$ genetic variance, so the new study was based on the premise that different approaches could provide new insight.

"Studies focusing on AD-related quantitative endophenotypes that manifest 15-20 years earlier than clinical $\mathrm{AD}$ provide a powerful alternative approach to identifying additional $\mathrm{AD}$-related genes and uncover mechanisms of $\mathrm{AD}$ and disease progression," says Kamboh.

The team focused on early $A \beta$ deposition in the brain and looked for genetic associations with this disease characteristic. The researchers combined data on $\mathrm{A} \beta$ deposition measured with Pittsburgh compound B (PiB) PET - in 1,000 individuals from three $\mathrm{AD}$ centres and the Alzheimer's Disease Neuroimaging Initiative. They then performed a meta-analysis of genome-wide association studies to identify loci associated with $\mathrm{A} \beta$ deposition.

Perhaps unsurprisingly, the $A P O E^{*} \varepsilon 4$ allele was the genetic factor most strongly associated with $A \beta$ deposition. However, the analysis provided new insight. "We showed that there are other variants in the $A P O E$ gene region that affect $A \beta$ deposition in the brain independently of $A P O E^{*} \varepsilon 4$," says Kamboh. "We have also identified novel regions other than APOE that affect $\mathrm{A} \beta$ deposition, but we need a larger sample size to demonstrate that these loci meet the threshold for genome-wide significance."

The team now plan to gather more PiB-PET data to enable a larger meta-analysis, to conduct whole-genome sequencing to map the novel loci identified, and to discover new gene variants that affect $A \beta$ deposition in the brain. They also plan to do similar work on tau biomarkers. "It is important to understand the complete genetic architecture of both amyloidosis and tau pathology to help to discover novel pathways and, eventually, drug targets," concludes Kamboh.

\section{Ian Fyfe}

ORIGINAL ARTICLES Sepulcre, J. et al. Neurogenetic contributions to amyloid beta and tau spreading in the human cortex. Nat. Med. https:// doi.org/10.1038/s41591-018-0206-4 (2018) | Yan, Q. et al. Genome-wide association study of brain amyloid deposition as measured by Pittsburgh Compound-B (PiB)-PET imaging. Mol. Psychiatry https://doi.org/10.1038/s41380-018-0246-7 (2018) 